\title{
Cardiofaciocutaneous Syndrome Phenotype in a Case with de novo KRAS Pathogenic Variant
}

\author{
Aslihan Sanri $^{\mathrm{a}}$ Hakan Gurkan ${ }^{\mathrm{b}}$ Selma Demir ${ }^{\mathrm{b}}$ \\ ${ }^{a}$ Department of Pediatric Genetics, Samsun Training and Research Hospital, Samsun, Turkey; ${ }^{b}$ Department of \\ Medical Genetics, Faculty of Medicine, Trakya University, Edirne, Turkey
}

\section{Established Facts}

- It is known that germline KRAS gene mutations have been linked to both Noonan syndrome and cardiofaciocutaneous syndrome phenotypes.

- KRAS T58I mutation has previously been described in a patient with Noonan syndrome.

\section{Novel Insights}

- Our report describes the connection between KRAS T58I mutation and cardiofaciocutaneous syndrome which has not been reported before.

\section{Keywords}

Cardiofaciocutaneous syndrome $\cdot$ KRAS · Noonan syndrome · RASopathies

\begin{abstract}
Cardiofaciocutaneous (CFC) syndrome is one of the developmental disorders caused by a dysregulation of the Ras/ mitogen-activated protein kinase (MAPK) pathway. RASopathies share overlapping clinical features, making the diagnosis challenging, especially in the newborn period. The majority of CFC syndrome cases arise by a mutation in the BRAF, $M A P 2 K 1, M A P 2 K 2$, or (rarely) KRAS genes. Germline KRAS mutations are identified in a minority of CFC and Noonan syndrome cases. Here, we describe a patient with a KRAS mutation presenting with a CFC syndrome phenotype. The female patient was referred for genetic testing because of con-
\end{abstract}

genital exophthalmos. Her facial appearance is distinctive with a coarse face, exophthalmos, ptosis, downslanting palpebral fissures, hypertelorism, deep philtrum, downturned corners of the mouth, and a short neck. She suffered from feeding difficulties, poor weight gain, and developmental delay. The sequencing of the genes involved in the MAPK pathway (PTPN11, SOS1, RAF1, KRAS, NRAS, MAP2K1, SHOC2, $C B L$, and SPRED1) identified a heterozygous de novo NM_004985.4:c.173C>T (p.Thr58lle) in the KRAS gene. Germline KRAS mutations have been identified in approximately $2 \%$ of the reported NS cases and less than $5 \%$ of the reported CFC syndrome cases. Because CFC and Noonan syndrome share clinical overlapping features, the phenotype caused by KRAS mutations is often difficult to assign to one of the 2 entities. The mutation that we detected in our patient was previously reported in a patient with an Noonan syndrome phenotype. However, our patient predominantly exhibits

\section{KARGER}

(C) 2019 S. Karger AG, Basel

E-Mail karger@karger.com

www.karger.com/msy
Aslihan Sanri

Department of Pediatric Genetics, Samsun Training and Research Hospital

Kadikoy, Barıs Blv. No.199

Ilkadım/Samsun 55090 (Turkey)

E-Mail aslihanaltunoglu@gmail.com 
CFC clinical features. In our case, coarse facial appearance and severe developmental delay help discriminate CFC from Noonan syndrome. Thus, patient follow-up, especially for delayed motor milestones suspected from RASopathies, is important for the discrimination of overlapping conditions as in the abovementioned syndromes.

(c) 2019 S. Karger AG, Basel

CFC syndrome belongs to a group of syndromes known as RASopathies, which are a class of developmental disorders caused by the dysregulation of the Ras/mitogen-activated protein kinase (MAPK) pathway [Tidyman and Rauen, 2009]. Ras/MAPK pathway disorders share overlapping clinical features, including craniofacial dysmorphism, hair and skin abnormalities, cardiac defects, cognitive impairment, and postnatal growth deficiency [Stark et al., 2012]. Although CFC syndrome has distinctive characteristics, many of its clinical features overlap with 2 other RASopathies, namely, Noonan syndrome and Costello syndrome, therefore making the diagnosis challenging, especially in the newborn period. CFC syndrome is an autosomal dominant disorder with the majority of cases arising from a mutation in the $B R A F$, $M A P 2 K 1, M A P 2 K 2$, or (rarely) KRAS genes [Abe et al., 2012]. Germline KRAS mutations are identified in a minority of patients with CFC and Noonan syndrome [Carta et al., 2006].

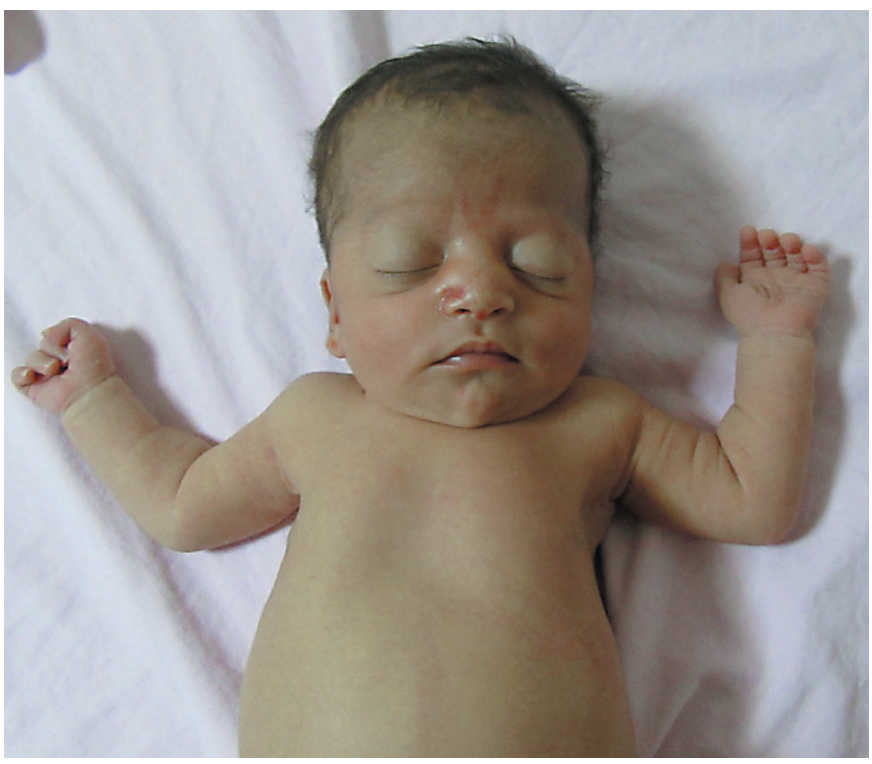

Fig. 1. Facial appearance of the patient at 17 days showing pronounced exopthalmous.

CFC Syndrome with de novo KRAS

Pathogenic Variant
We describe a patient with KRAS T58I mutation presenting with a CFC syndrome phenotype.

\section{Case Report}

A 17-day-old girl was referred to the genetics department because of congenital exophthalmos. She is the first child of a healthy nonconsanguineous couple. During pregnancy, fetal nuchal edema was recorded. She was born at 36 weeks of gestation, with a birth weight of $3 \mathrm{~kg}$ (50-75th percentile), length of $45 \mathrm{~cm}$ (10-25th percentile), and a head circumference of $34 \mathrm{~cm}$ (5075 th percentile). The family history was negative for known genetic disorders. Her facial appearance is distinctive with bitemporal narrowing, exophthalmos, ptosis, downslanting palpebral fissures, depressed nasal root, hypertelorism, anteverted nares, high-arched palate, a deep philtrum, downturned corners of the mouth, retrognathia, and a short neck (Fig. 1). Echocardiogram showed a secundum-type atrial septal defect. Hearing assessment and eye examination were normal except for exophthalmos and ptosis. Thyroid function tests were in a normal range. Abdominal ultrasonography revealed a hemangioma in the liver. Brain imaging was normal. During reexamination at the age of 5 months, her weight was $4.6 \mathrm{~kg}$ (3rd percentile), height was $59 \mathrm{~cm}(<3 \mathrm{rd}$ percentile), and head circumference was $40.5 \mathrm{~cm}$ (10-25th percentile). She also had a coarse face, relative macrocephaly, nystagmus, and mild pectus excavatum deformity (Fig. 2). She suffered from feeding problems and poor weight gain. She was able to hold her head independently at 4 months. At the age of 1 year, her coarse face became more prominent, and her feeding problems were unresolved. She could not sit without support and had delayed speech development. At age of 17 months, she was able to sit independently.

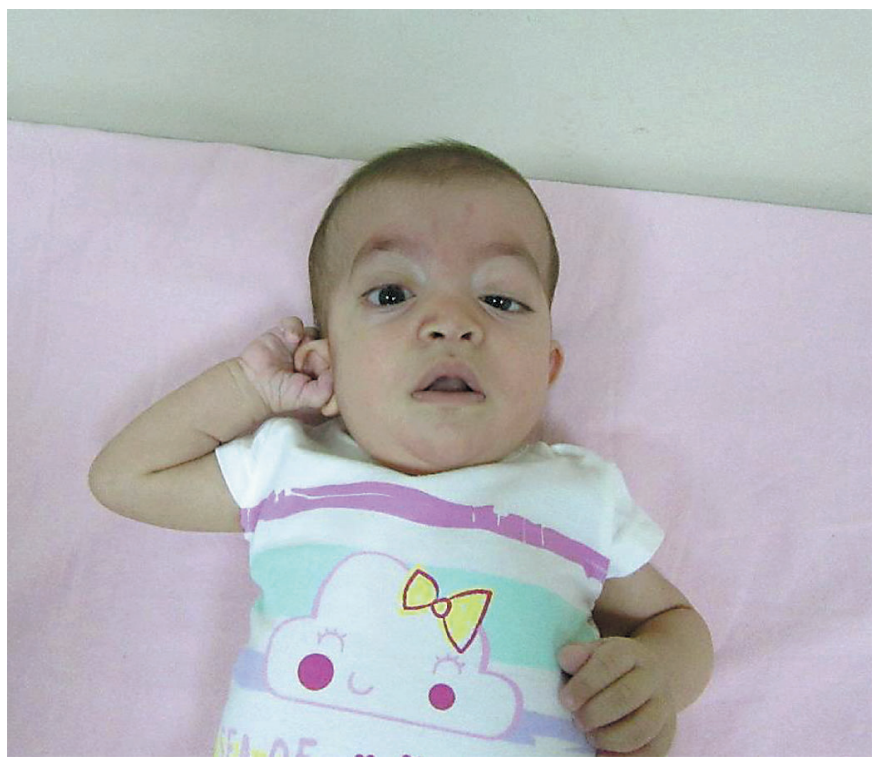

Fig. 2. Facial appearance of the patient at 5 months showing coarse facial features. 


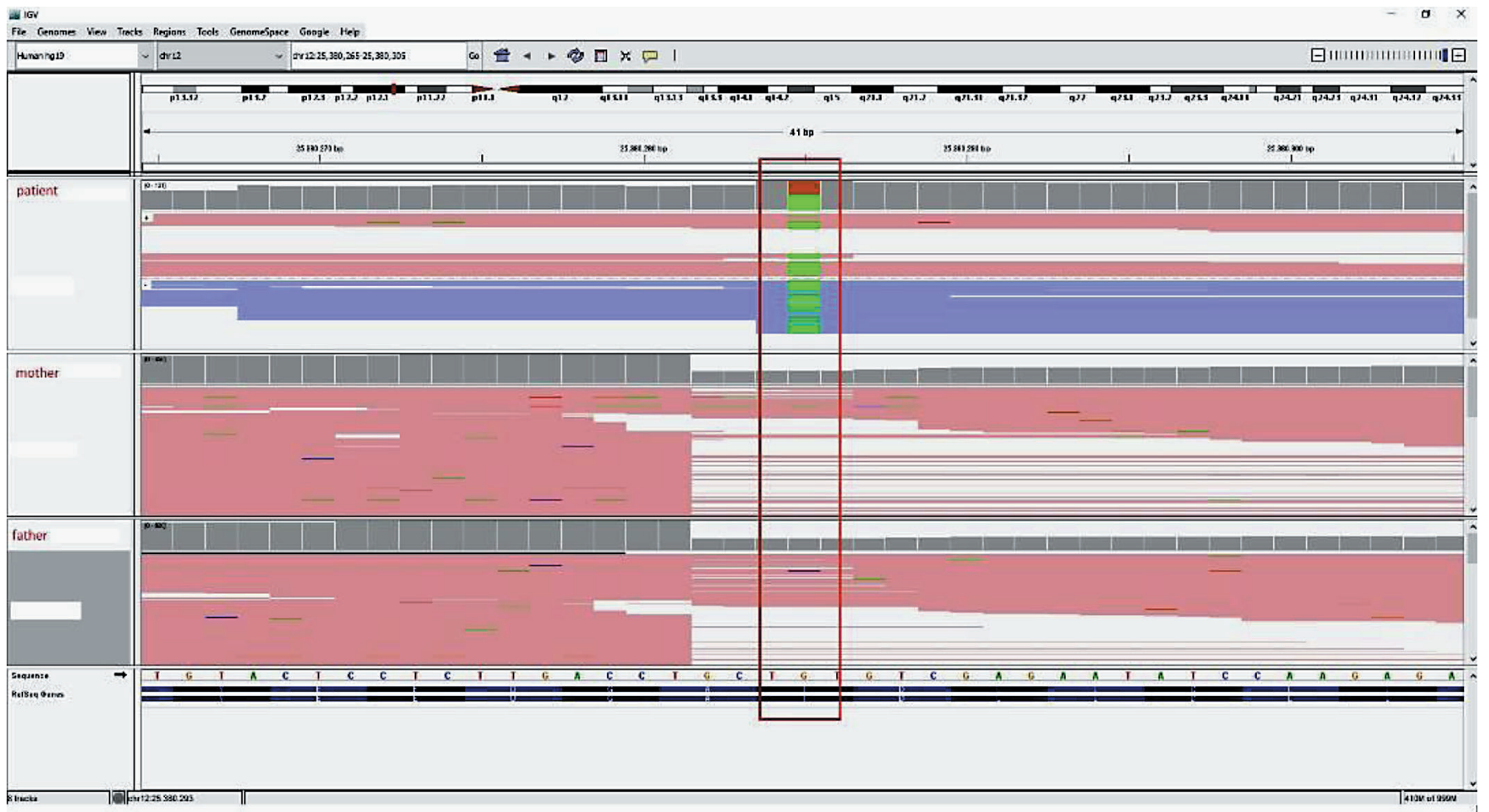

Fig. 3. IGV data of the patient and the parents, revealing the de novo origin of the heterozygous NM_004985.4:c.173C>T (p.Thr58Ile) variant.

\section{Materials and Methods}

Genomic DNA was isolated from nucleated peripheral blood cells. Libraries of the coding sequences of the genes of interest (PTPN11, SOS1, RAF1, KRAS, NRAS, MEK1, MEK2, SHOC2, CBL, and SPRED1) were prepared using the SG amplicon kits (Systemas Genomicos, Spain) and sequenced in the Illumina MiSeQ system. Illumina MiSeq Software was used to analyze the data, Genomize Seq (Genomize, Turkey) to filter the variants, and the IGV_2.3.8 program to evaluate the data visually.

\section{Results}

The sequencing revealed a heterozygous pathogenic NM_004985.4:c.173C > T (p.Thr58Ile) variant in the patient. The segregation analysis showed that this pathogenic variant was not present in parental DNA, confirming its de novo origin in the patient (Fig. 3).

\section{Discussion}

In the KRAS gene of our patient we identified a heterozygous de novo NM_004985.4:c.173C $>$ T (p.Thr58Ile). KRAS is a key component of the Ras/MAPK pathway, and heterozygous germline KRAS mutations have been identified in approximately $2 \%$ of the reported Noonan syndrome cases and less than $5 \%$ of the reported CFC syndrome cases [Carta et al., 2006; Schubbert et al., 2006]. Ras isoforms encoded by KRAS, HRAS, and NRAS represent highly conserved signal transduction molecules. Ras proteins act as molecular switches by cycling between active guanosine- $5^{\prime}$-triphosphate (GTP)-bound and inactive guanosine diphosphate-bound conformations; in their active form, they interact with a variety of downstream effector proteins. The majority of somatic Ras gene mutations found in various types of human cancer cause Ras to accumulate in the active GTP-bound state by impairing intrinsic GTPase activities and conferring resistance to GTPase-activating proteins. Germline KRAS mutations associated with Noonan/CFC syndrome are distinct from those found in malignancies and may give rise to mutant proteins with a relatively mild gain of function, which are tolerated in the germline and during embryonic development. The germline KRAS T58I mutation was first described in a patient with severe Noonan syndrome [Schubbert et al., 2006; Zenker et al., 2007]. The T58I mutation was considered to be pathogenic because of the de novo occurrence. The amino acid ex- 
change was predicted to affect highly conserved residues, which are not found in a normal control population, but appear in databases as a single-nucleotide polymorphism. In the same report, KRAS T58I showed a reduced intrinsic GTPase activity relative to wild-type KRAS, and this reduced activity may explain the severe clinical phenotype [Schubbert et al., 2006]. Because CFC and Noonan syndrome share clinical overlapping features, the phenotype caused by KRAS mutations is often difficult to assign to one of the 2 entities [Zenker et al., 2007]. The discrimination between Noonan, Costello, and CFC syndrome, however, is possibly based on distinct clinical features such as dry hyperkeratotic skin, hair abnormalities in patients with CFC syndrome, redundant and loose skin with deep palmar and plantar creases in those with Costello syndrome, and coarse facial appearance as well as severely impaired mental development in patients with CFC and Costello syndrome. Noonan syndrome is usually associated with minor cognitive deficits or even normal intelligence [Allanson, 1987; Rodriguez-Viciana et al., 2006]. The mutation we detected in our patient was previously reported in a patient with an Noonan syndrome phenotype [Schubbert et al., 2006]. This patient presented with Noonan-like facial features, developmental delay, and myeloproliferative disorders, such as juvenile myelomonocytic leukemia. However, our patient predominantly exhibits CFC clinical features with a coarse facies. During the follow-up, her facial appearance worsened and her motor milestones were severely delayed. Here, we present a patient with congenital exophthalmos which is a very rare manifestation in neonates. A variety of malignant neoplastic, benign neoplastic, cystic, and vascular etiologies have been associated with proptosis at birth [Erickson and Tse, 2014]. The ophthalmological examination of the patient was normal, except for exophthalmos and ptosis. Brain imaging ruled out orbital and cranial lesions, and an intra-abdominal mass (hemangioma) was detected via ultrasonography. Proptosis is one of the ocular features of CFC syndrome, but only a few reports with congenital presentation are available [Manci et al., 2005; Rodriguez-Viciana et al., 2006].

Regardless of the phenotypic assignment, establishing the molecular etiology of all individuals with CFC syndrome is essential to provide appropriate medical care. In addition, molecular analysis is important for genetic counseling and the understanding of recurrence risk. Thus, patient follow-up, especially for delayed motor milestones suspected from RASopathies, is important for the discrimination of overlapping conditions such as CFC, Noonan, and Costello syndrome.

\section{Statement of Ethics}

The authors have no ethical conflicts to declare.

\section{Disclosure Statement}

The authors have no conflicts of interest to disclose.

\section{References}

-Abe Y, Aoki Y, Kuriyama S, Kawame H, Okamoto N, et al: Costello and CFC syndrome study group in Japan. Prevalence and clinical features of Costello syndrome and cardio-faciocutaneous syndrome in Japan: findings from a nationwide epidemiological survey. Am J Med Genet A 158A:1083-1094 (2012).

Allanson JE: Noonan syndrome. J Med Genet 24: 9-13 (1987).

Carta C, Pantaleoni F, Bocchinfuso G, Stella L, Vasta I, et al: Germline missense mutations affecting KRAS isoform $B$ are associated with a severe Noonan syndrome phenotype. Am J Hum Genet 79:129-135 (2006).
Erickson BP, Tse DT: Management of neonatal proptosis: a systematic review. Surv Ophthalmol 59:378-392 (2014).

Manci EA, Martinez JE, Horenstein MG, Gardner TM, Ahmed A, et al: Cardiofaciocutaneous syndrome (CFC) with congenital peripheral neuropathy and nonorganic malnutrition: an autopsy study. Am J Med Genet 137:1-8 (2005).

Rodriguez-Viciana P, Tetsu O, Tidyman WE, Estep AL, Conger BA, et al: Germline mutations in genes within the MAPK pathway cause cardiofaciocutaneous syndrome. Science 311: 1287-1290 (2006)
Schubbert S, Zenker M, Rowe SL, Böll S, Klein C, et al: Germline KRAS mutations cause Noonan syndrome. Nat Genet 38:331-336 (2006).

-Stark Z, Gillessen-Kaesbach G, Ryan MM, Cirstea IC, Gremer L, et al: Two novel germline KRAS mutations: expanding the molecular and clinical phenotype. Clin Genet 81:590-594 (2012).

Tidyman WE, Rauen KA: The RASopathies: developmental syndromes of Ras/MAPK pathway dysregulation. Curr Opin Genet Dev 19: 230-236 (2009).

Zenker M, Lehmann K, Schulz AL, Barth H, Hansmann D, et al: Expansion of the genotypic and phenotypic spectrum in patients with KRAS germline mutations. J Med Genet 44:131-135 (2007).
CFC Syndrome with de novo KRAS

Pathogenic Variant
Mol Syndromol 2019;10:344-347 DOI: $10.1159 / 000504374$ 